\title{
Métodos participativos y construcción con tierra en barrios urbanos pobres de Argentina
}

\author{
Participatory methods and earthen construction \\ in urban slums of Argentina
}

Rodolfo Rotondaro* y Fernando Cacopardo***

Citar este artículo como: Rotondaro, R. y Cacopardo, F. (2019). Métodos participativos y construcción con tierra en barrios urbanos pobres de Argentina. Revista Nodo, 14(27), pp. 38-52

\section{Resumen}

El propósito de esta investigación es el desarrollo de métodos participativos para generar soluciones constructivas para el hábitat de contextos urbanos pobres en Argentina. Los objetivos se inscriben en el marco conceptual de la pobreza urbana y las tecnologías sociales. El área de estudio incluye a barrios de las ciudades de Mar del Plata y Buenos Aires cuya población mantiene una condición de pobreza estructural. La metodología está focalizada en la práctica de métodos participativos para generar nuevos materiales, morteros y elementos constructivos con suelos estabilizados, y su adecuada apropiación por parte de los autoconstructores locales. Se introdujeron innovaciones que los combinan con materiales, elementos y técnicas propias de la construcción tradicional industrializada, habituales en los barrios. Participan vecinos y sus familias, técnicos, profesionales, estudiantes, voluntarios, empresas privadas, municipalidades y ONGs. Se construyeron prototipos de pisos, muros, revoques, hornos y estufas, que fueron analizados y evaluados en territorio en relación a sus características técnicas, de aceptación y de producción. Los principales resultados indican que los prototipos de mejor proyección para su desarrollo fueron aquellos más cercanos a las lógicas de la autoconstrucción y a las particularidades de cada grupo familiar en cada barrio.

Palabras clave: tecnología social, vivienda, construcción con tierra.

\section{Abstract}

The aim of this research is the development of participative methods and alternative earth building materials and elements for housing in the poor urban contexts of Argentina. The urban poverty and the Social Technology theories were been considered to define the objectives.

Fecha de recepción: 15 de abril de 2019 - Fecha de aceptación: 16 de agosto de 2019

\footnotetext{
* Arquitecto y Magister CEAA del Centro Internacional de la Construcción con Tierra, CRATerre-EAG, Francia. Universidad de Buenos Aires -CONICET. Facultad de Arquitectura, Diseño y Urbanismo, Instituto de Arte Americano e Investigaciones Estéticas, Buenos Aires, Argentina. Correo electrónico: rodolforotondaro@gmail.com

*: Arquitecto y Magister en Historia, Universidad Nacional de Mar del Plata-CONICET. Facultad de Arquitectura, Urbanismo y Diseño. Instituto de Investigaciones, Desarrollo Urbano, Tecnología y Vivienda, Mar del Plata, Provincia de Buenos Aires, Argentina. Correo electrónico: ferjupiter@yahoo.com
} 
Fieldwork was developed with the participation of the vulnerable population in neighborhoods of Mar del Plata and Buenos Aires's cities. The methodology is focused on the participative management methods to generate the new elements into the emergency contexts, and the appropriation by the local builders. Are introduced technical innovations based on the use of natural materials and elements with stabilized soils, and its combination with conventional industrial building materials, elements, and techniques. Are involved self builders, technicians, professionals, students, volunteers, enterprises, municipalities, and NGOs. Are built and studied different prototypes of alternative floors, walls, plasters and domiciliary stoves using earth building techniques and mixed building techniques.

Keywords: social technology, housing, earthen construction.

\section{Introducción}

\section{Pobreza urbana y tecnologías sociales}

El marco teórico-conceptual de esta investigación está basado en las complejidades de la pobreza urbana (Pelli, 1994; Kessler y Di Virgilio, 2008, p. 32 ), situación que afecta a las ciudades medias y grandes de Argentina; y en la teoría de las tecnologías sociales (Dagnino et al., 2004; Thomas, 2009). La pobreza urbana ha crecido en las últimas tres décadas en Argentina, especialmente en la periferia de las principales ciudades. Es el caso del área metropolitana conocida como Gran Buenos Aires (GBA), y las ciudades de tamaño medio, tales como Mar del Plata-Batán, en la provincia de Buenos Aires.

La information estadística censal oficial (Tabla 1) muestra los valores de los sectores urbanos en situación de pobreza de estas ciudades, pobreza que afecta de manera considerable las condiciones de calidad de vida de sus habitantes.

Tabla 1. Información y análisis estadístico de Población y Vivienda del conglomerado del Gran Buenos Aires y de la ciudad de Mar del Plata-Batán, según los últimos dos censos nacionales del Instituto Nacional de Estadística y Censos (INDEC, 2002; 2011). Porcentajes comparados con los valores totales de Argentina.

\begin{tabular}{|l|c|c|c|}
\hline & Argentina & $\begin{array}{c}\text { Gran Buenos Aires } \\
\text { (Ciudad de Buenos } \\
\text { Aires y 24 estados)* }\end{array}$ & $\begin{array}{c}\text { Ciudad de Mar del } \\
\text { Plata-Batán }\end{array}$ \\
\hline Población total & $40,117,096$ & $9,916,715(24.72 \%)$ & $618,898(1.55 \%)$ \\
\hline Número total Hogares & $39,675,905$ & $2,934,373(7.40 \%)$ & $209,794(0.53 \%)$ \\
\hline Población bajo la Línea de Pobreza (LP) & & & $25,375(2.14 \%)$ \\
-Hogares & $1,189,000$ & $87,986(7.40 \%)$ & $33,420(0.75 \%)$ \\
-Población & $4,480,000$ & $1,107,456(24.72 \%)$ & $25,375(2.14 \%)$ \\
\hline Población bajo la Línea de Indigencia (LI) & & & $13,616(3.71 \%)$ \\
-Hogares & 189,000 & $13,986(7.40 \%)$ & $90,772(24.72 \%)$ \\
-Población & 367,000 & & \\
\hline
\end{tabular}

Fuente: Elaboración propia, a partir de la información del Instituto Nacional de Estadística y Censos (INDEC, 2013): Censo Nacional de Población, Hogares y Viviendas 2001 y 2010; Encuesta Permanente de Hogares).

* Septiembre 2013: 24 estados (Almirante Brown, Avellaneda, Berazategui, Esteban Echeverría, Ezeiza, Florencio Varela, General San Martín, Hurlingham, Ituzaingó, José C. Paz, La Matanza, Lanús, Lomas de Zamora, Malvinas Argentinas, Merlo, Moreno, Morón, Quilmes, San Fernando, San Isidro, San Miguel, Tigre, Tres de Febrero, Vicente López).

** Diciembre de 2012. 
La crisis de los años 2001 y 2002 ha contribuido a consolidar la pobreza estructural en el país, en general, rural y urbana. En esos años, la Línea de Pobreza (LP) alcanzó alarmantes porcentajes del $57.7 \%$ y $27.5 \%$ de la población en condiciones de indigencia (INDEC, 2002). Sin embargo, la economía del país ha cambiado en los años recientes. En 2008 los niveles de pobreza de la población urbana se mantuvo en el $17.8 \%$ durante la primera mitad del año, y en 2009 un poco más de 4 millones de argentinos no tenía satisfechas sus mínimas necesidades de calidad de vida.

En 2011, según la información del INDEC, el porcentaje de pobreza en el país era del $22 \%$, y en el Gran Buenos Aires era del 8.4\%. Sin embargo, consultores privados estimaban que en 2012 un $26 \%$ de la población estaba bajo la Línea de Pobreza.

Aún considerando las diferencias entre la información pública y la privada, la pobreza estructural así como la indigencia, el sub-empleo y la inseguridad en el sector laboral, son la realidad de los problemas cotidianos de miles de personas en las principales ciudades del país. El deterioro social y de vivienda en estas áreas urbanas es crítico, y permanente durante décadas, y la asistencia del gobierno no sólo es insuficiente sino inadecuada.

\section{Área de estudio y marco institucional}

Las áreas donde se trabaja incluyen a territorios de la periferia de las dos ciudades mencionadas, en el centro de Argentina (Figura 1). Una de ellas es Mar del Plata-Batán, una ciudad de mediana escala en tamaño y población; la otra es la metrópolis de Buenos Aires, conglomerado de más de 14 millones de habitantes y numerosos y variados asentamientos informales y villas miseria.

En Mar del Plata se trabajó principalmente en tres barrios de la periferia: Monte Terrabusi, Alto Camet y Nuevo Golf. En el Conurbano de Buenos Aires se incluyeron dos barrios: Bancalari, en

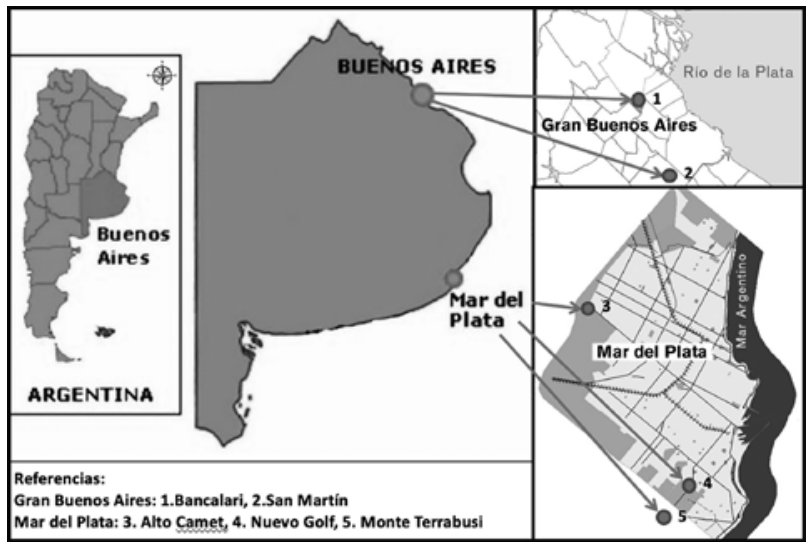

Figura 1. Localización de las áreas de trabajo en el Gran Buenos Aires y en la ciudad de Mar del Plata. Fuente: elaboración propia.

el departamento de Tigre; y Loma Hermosa, en el departamento de San Martín. Los grupos de trabajo están integrados por investigadores, becarios, alumnos de grado y voluntarios, cuyos programas de investigación tienen sede en dos universidades estatales: la Facultad de Arquitectura, Diseño y Urbanismo de la Universidad de Buenos Aires; y la Facultad de Arquitectura, Urbanismo y Diseño de la Universidad Nacional de Mar del Plata.

Varios organismos y entidades públicas y privadas brindaron de manera parcial, soporte material, financiero y de infraestructura. Los principales son los siguientes: la Municipalidad de General Pueyrredón; Municipalidad de Tigre; el gobierno de la Provincia de Buenos Aires; el Consejo Nacional de Investigaciones Científicas y Tecnológicas (CONICET); la Fundación SOPORTE, de Mar del Plata; otros organismos no gubernamentales; y varias empresas públicas y privadas que donan servicios técnicos y materiales de construcción (Cantera Yaraví, empresa de electricidad EDEA, ONG CEAS, ONG SEDECA).

\section{Objetivos de la investigación}

La investigación tiene como principales objetivos diseñar y experimentar nuevas estrategias para el mejoramiento de la vivienda y el hábitat de grupos 
familiares en contextos urbanos pobres, teniendo en cuenta el marco teórico de las tecnologías sociales y el empleo de materiales y técnicas constructivas sustentables (con énfasis en el empleo de suelos estabilizados y de técnicas de la construcción con tierra).

Los desarrollos tecnológicos y las innovaciones son pensadas a partir de las características específicas de cada grupo familiar seleccionado, y teniendo en cuenta el entorno de cada vivienda, las dinámicas socio-culturales y la disponibilidad de recursos humanos y materiales en cada lugar.

Las formas de intervención en los territorios contemplan métodos alternativos no convencionales de generación de tecnología, posibles de aplicar en estos contextos urbanos, y promueven acciones que puedan contribuir con la producción de mejoras de baja escala en el campo del habitat en general, y de la vivienda en particular (Pelli, 2007, pp. 65-81).

\section{Metodología}

El trabajo principal se realizó en cada barrio junto con los vecinos interesados en participar, en base a los mapas de riesgo y a los indicadores de vulnerabilidad más alta, con la coordinación del grupo de investigadores (tanto en Mar del Plata como en Buenos Aires). La participación de otros actores externos a los barrios, tales como las ONGs, las empresas privadas, los organismos estatales y los grupos de pasantes y voluntarios universitarios, pudo realizarse a partir de la construcción de alianzas socio-técnicas con objetivos específicos y acciones precisas, según el diagnóstico de necesidades y prioridades de las familias, así como también de los recursos obtenidos para cada caso.

Es muy importante para la investigación en curso el diseño de métodos participativos ajustados localmente para poder construir alianzas: por un lado, entre los vecinos y sus organizaciones y el resto de los actores públicos y privados; y por otro lado, entre estos últimos.
De acuerdo con la estrategia y los objetivos mencionados anteriormente, la tecnología de construcción en experimentación impulsa el uso de materiales, morteros y elementos de la construcción con tierra para promocionar el empleo de materiales sustentables, que contribuyan con el ahorro energético y a disminuir la contaminación ambiental, y que fomenten además la participación de autoconstructores en prácticas comunitarias organizadas.

El diseño de los prototipos se realizó como parte de un proceso que se trata de sostener en el tiempo, y que se basa en la combinación del saberhacer de los autoconstructores de los barrios, y sus lógicas constructivas en la materialización de sus viviendas y su hábitat doméstico, con el conocimiento técnico-científico de los investigadores, con el único fin de generar mejoras progresivas y a baja escala en el contexto de cada barrio.

La tecnología de la construcción con tierra se combinó con otras tecnologías de la autoconstrucción popular, que habitualmente emplea materiales y elementos industrializados, de tal modo que los prototipos alternativos coexisten con los modos propios de la autoconstrucción popular. Se busca generar mejoras simples, familia por familia, accesibles en costos y esfuerzo. Es decir: posibles de desarrollar y replicar en los territorios reales.

Algunas de las técnicas de construcción con tierra conocidas fueron seleccionadas y son la base del diseño de los prototipos, con la adaptación más apropiada según la disponibilidad de personas, de tiempo y de recursos materiales del lugar.

Los cambios y ajustes de las acciones que se realizaron en el marco de cada actividad y desarrollo tecnológico fueron discutidos entre los beneficiarios de cada barrio y el grupo de investigación, para arribar a decisiones por consenso de mayoría. Las alianzas también se fueron modificando y adaptando de acuerdo con los aciertos y las dificultades que se presentaron en cada caso. Se realizaron nuevos acuerdos en la medida que 
fueron solicitados, o bien en aquellos casos en que los acuerdos existentes se consolidaron o convergieron en una escala de mayor complejidad.

En la selección de materiales se tuvieron en cuenta los suelos naturales existentes y disponibles en cada barrio, como material de base, para modificar según el tipo y la función del componente constructivo. Esta modificación incluyó desde el tamizado mediante tamices metálicos rústicos, hasta el mezclado con los estabilizantes habitualmente empleados, naturales e industrializados: fibras vegetales naturales (paja), arena, piedra, escombros de obra, cal hidráulica, cemento Porland.

Se realizaron análisis cualitativos y sensoriales de los suelos y las fibras locales disponibles, con la realización de algunos talleres de capacitación en los barrios. Estas acciones fueron útiles para explorar las capacidades y las expectativas de los autoconstructores y vecinos en general. Los ensayos sensoriales incluyeron tests de textura, sedimentación simple, plasticidad, resistencia en seco y retracción lineal (Neves et al., 2009, pp.1526).

Para la elaboración de los morteros y mezclas de relleno se utilizaron materiales naturales e industriales, tales como suelos arcillosos y suelos arenosos, arena, fibras vegetales naturales, agua, cal hidratada y cemento Portland. Para los elementos portantes en muros se trabajó con postes rollizos, madera de pallets usados, vigas y columnas de madera y metálicas con chapa doblada de hierro galvanizado, alambre negro, clavos y otros elementos metálicos de sujeción.

En la construcción de los prototipos y de todas las pruebas de materiales y morteros realizadas en los barrios se emplearon herramientas de la construcción tradicional y equipos adquiridos o prestados (tales como tamices, las dos prensas de los bloques de tierra comprimida (BTC), mezcladoras mecánicas, moldes para tierra vertida), de acuerdo con el tipo de elemento o proceso de producción.

\section{Gestión, diseño y construcción de prototipos en el territorio}

En las dos ciudades se construyeron diferentes prototipos de piso, contrapiso, murode cerramiento vertical, revoques y terminaciones, estufa y hornos domiciliarios con paredes de adobe. Se emplearon adobes, BTC, pallets reciclados, morteros de asiento de suelos naturales estabilizados con fibras naturales, morteros de suelo-cemento, morteros mixtos, revoques de suelo natural estabilizado con cemento y cal, baldosas de suelo-cemento, ladrillos refractarios y elementos metálicos.

Se describen a continuación las principales características de varios de los prototipos construidos en distintos barrios, con formas de gestión y actores que surgieron en cada contexto:

\section{A. Contrapisos y pisos alternativos en viviendas y un centro vecinal (Figura 2 , Tabla 2)}

En Bancalari y San Martín (GBA) y en Monte Terrabusi (Mar del Plata) se construyeron varios prototipos de contrapiso y piso, adaptando suelos y recursos materiales locales (Rotondaro, Patrone y Schicht, 2006, pp. 56-57).

Los contrapisos se materializaron con una o dos capas sucesivas de morteros mixtos compuestos por suelo natural del lugar sin tamizar, cascotes de ladrillo, arena, cal hidratada, cemento Portland y agua, variando los porcentajes en volumen del aglomerante industrial (cemento o cal hidratada) y de los agregados gruesos. Los morteros se prepararon con mezclado manual y con mezcladora mecánica, hasta obtener la plasticidad necesaria para permitir su manipuleo y colado.

La técnica constructiva fue la habitual utilizada en la autoconstrucción: colado del mortero sobre el sector de piso natural preparado con nivelación, apisonado y un polietileno de 200 micrones como aislante. Los espesores totales de los contrapisos construidos varían entre 11 y $15 \mathrm{~cm}$. 


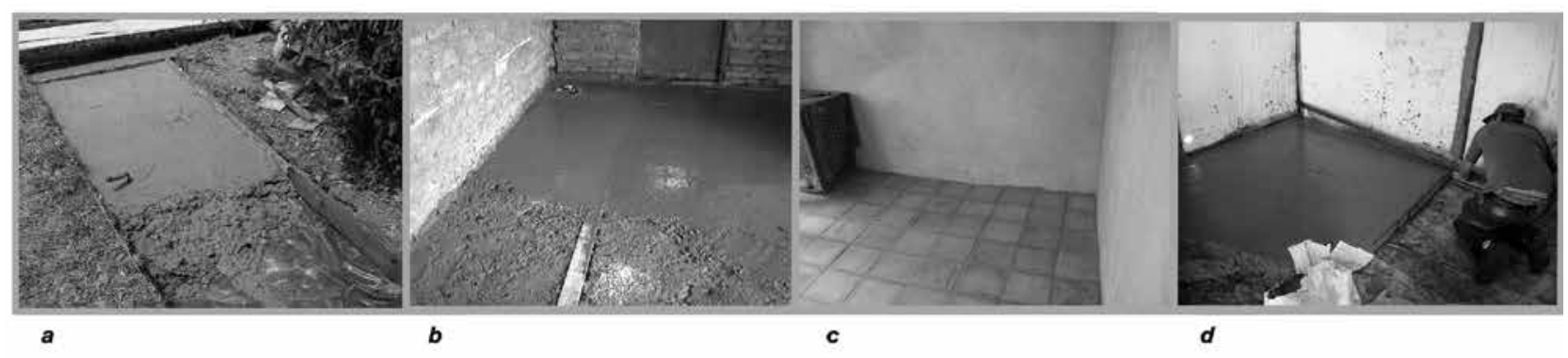

Figura 2. Prototipos de pisos en Bancalari (a, b) y San Martín (c), en Buenos Aires; y en Nuevo Golf (d) en Mar del Plata. Fuente: Rodolfo Rotondaro.

Tabla 2. Características técnicas de los prototipos de contrapisos y pisos.

\begin{tabular}{|c|c|c|c|c|c|}
\hline Prototipo & $\begin{array}{l}\text { Espesor } \\
(\mathrm{cm})\end{array}$ & $\begin{array}{l}\text { Materiales/ } \\
\text { morteros* }\end{array}$ & Técnica & $\begin{array}{l}\text { Diseñadores/ } \\
\text { constructores }\end{array}$ & Otros aspectos \\
\hline $\begin{array}{l}\text { Contrapiso } \\
\text { bicapa en } \\
\text { Bancalari (a) }\end{array}$ & $\begin{array}{l}14,5 \\
0,5 \\
\text { (capa final) }\end{array}$ & $\begin{array}{l}8: 4: 2: 1: 1 / 4 \\
\text { scombros-suelos- } \\
\text { arena-cal-cemento }\end{array}$ & $\begin{array}{l}\text { Colado sobre } \\
\text { polietileno } \\
\text { aislante alisado a } \\
\text { llana }\end{array}$ & $\begin{array}{l}\text { Vecinos, } \\
\text { arquitectos, } \\
\text { estudiantes }\end{array}$ & $\begin{array}{l}\text {-Construcción rápida } \\
\text {-Posible para los } \\
\text { autoconstructores }\end{array}$ \\
\hline $\begin{array}{l}\text { Baldosas } \\
\text { prefabricadas } \\
\text { en Bancalari } \\
\text { (b) }\end{array}$ & $\begin{array}{l}2 \\
\text { (base) } \\
0,5 \\
\text { (capa final) }\end{array}$ & $\begin{array}{l}8: 2: 1 / 2 * * \\
\text { suelos-arena- } \\
\text { cemento } \\
1: 1: 3 \\
\text { suelo-cemento-arena }\end{array}$ & $\begin{array}{l}\text { Colado en moldes } \\
\text { de madera y } \\
\text { metálicos alisado } \\
\text { con fratacho y } \\
\text { llana }\end{array}$ & $\begin{array}{l}\text { Vecinos, } \\
\text { arquitectos, } \\
\text { estudiantes }\end{array}$ & $\begin{array}{l}\text {-Posible para los } \\
\text { autoconstructores } \\
\text {-Necesita más tiempo } \\
\text { de fabricación que } \\
\text { los contrapisos/pisos } \\
\text { colados }\end{array}$ \\
\hline $\begin{array}{l}\text { Contrapiso/ } \\
\text { piso en San } \\
\text { Martín (c) }\end{array}$ & $\begin{array}{l}12 / 14 \\
0,2 \\
\text { (alisado } \\
\text { final) }\end{array}$ & $\begin{array}{l}: 6: 2: 1 / 2 \\
\text { escombros-suelos- } \\
\text { cal-cemento } \\
1: 1: 3 \\
\text { suelo-cemento-arena }\end{array}$ & $\begin{array}{l}\text { Colado sobre } \\
\text { terreno natural } \\
\text { nivelado } \\
\text { Apisonado alisado } \\
\text { con regla y llana }\end{array}$ & $\begin{array}{l}\text { Vecinos, } \\
\text { arquitectos, } \\
\text { estudiantes }\end{array}$ & $\begin{array}{l}\text {-Construcción rápida } \\
\text {-Posible para los } \\
\text { autoconstructores }\end{array}$ \\
\hline $\begin{array}{l}\text { Contrapiso/ } \\
\text { piso en Nuevo } \\
\text { Golf }(d)\end{array}$ & 11 & $\begin{array}{l}8: 6: 2: 1 / 2: 1 / 4 \\
\text { escombros-suelos- } \\
\text { arena-cal-cemento }\end{array}$ & $\begin{array}{l}\text { Relleno y alisado } \\
\text { superficial }\end{array}$ & $\begin{array}{l}\text { Vecinos, } \\
\text { arquitectos, } \\
\text { estudiantes }\end{array}$ & $\begin{array}{l}\text {-Construcción rápida } \\
\text {-Posible para los } \\
\text { autoconstructores }\end{array}$ \\
\hline
\end{tabular}

"Porcentaje de partes en volumen.

** Representa uno de los porcentajes de materiales en volumen de modelos de baldosas que obtuvieron mejores resultados de resistencia mecánica a la rotura por flexión y a la abrasión (capa superficial).

Sobre estos contrapisos se experimentaron dos soluciones constructivas de terminación (piso o solado): una carpeta alisada de base cementicia, y baldosas prefabricadas. En el caso de las carpetas, se construyeron con morteros de arena, cemento y agua, probando dos porcentajes de cemento en volumen, en una capa de aproximadamente 0,5 a $0,6 \mathrm{~cm}$ de espesor. Se colocó con ayuda de regla de madera y se finalizó con un espolvoreado de cemento puro después del primer secado, a las dos horas.
En el caso de las baldosas, su dimensión es cuadrada, de $25 \mathrm{~cm}$ de lado y 2,5 $\mathrm{cm}$ de espesor. Se fabricaron con moldes de madera y metálicos, con un lado desmontable, usando como desmoldante aceite usado, en dos capas: la primera de 2 $\mathrm{cm}$ de espesor, con suelo-cemento colado y alisado con fratacho; la segunda de $0,5 \mathrm{~cm}$ de espesor, con cemento: arena 1:2 y 1:3 la mayoría de los modelos, alisada con llana y finalizada con espolvoreado de cemento puro. 


\section{B. Muros de mampostería de bloques de tierra comprimida (BTC), de entramado con pallets y colado in situ de tierra vertida (Figura 3, Tabla 3)}

Los prototipos de muro construidos en Bancalari, Monte Terrabusi y Nuevo Golf (Rotondaro y Cacopardo, 2013, pp. 17-22) incluyeron distintas técnicas: a) con BTC, fabricados con dos tipos de prensas, una manual y otra hidráulica; b) un sistema de muro con técnica mixta que combina pallets de madera reciclados con morteros de suelos y fibras naturales; y un sistema in situ de tierra vertida (Aranda Jiménez y Roux Gutiérrez, 2012, pp. 115-122), con un mortero mixto colado en encofrado móvil.

En el caso de los BTC, los materiales empleados fueron suelos locales tamizados con malla de entre 5 y $8 \mathrm{~mm}$ de abertura, cemento tipo Portland y agua limpia. Se probaron dos morteros con diferente porcentaje en volumen de cemento: $7 \mathrm{y}$ $10 \%$, de los cuales se adoptó el mayor porcentaje para la fabricación en serie.

La técnica de fabricación fue la habitual utilizada para BTC estabilizados con cemento tipo Portland: mezclado en seco de los suelos con el cemento hasta obtener color uniforme, humedecido, prueba manual de humedad de compactación, prensado, colocación en tarimas en pilas cruzadas aireadas, curado de una semana y secado de tres semanas más. Una de las prensas es del tipo CINVA-RAM, de compresión simple, y fue proporcionada por un vecino de Monte Terrabusi (BTC de 9,6 cm x 14 cm x $29 \mathrm{~cm}$ ). La otra prensa pertenece a la Fundación UOCRA de Mar del Plata (Unión Obrera de la Construcción de la República Argentina), es hidráulica de doble compresión y matriz para bloque de intertraba (de $11 \mathrm{~cm} \times 18 \mathrm{~cm} \times 22 \mathrm{~cm}$ ). Estos BTC se fabricaron y secaron en instalaciones propias de la UOCRA, luego trasladados a Monte Terrabusi.

En el caso de los muros con técnica mixta, se construyeron en una vivienda rectangular de dos ambientes, con estructura independiente de rollizos de eucalipto y techo liviano de madera, membrana aislante y chapa de cinc. Con los pallets y algunas vigas menores de madera se construyeron los paños de muro superponiendo los pallets en vertical, sujetados con bulones a la estructura y entre sí. El relleno del muro se realizó con un mortero fabricado in situ con suelos del terreno de la obra y fibra natural picada obtenida en terrenos vecinos, del mismo modo que el revoque grueso, aplicado sobre una malla metálica clavada a los pallets.

Sobre los pallets se clavó una malla metálica de gallinero sobre la cual se aplicó una capa de $2,5 \mathrm{~cm}$ de espesor promedio de revoque grueso, confeccionado en pisadero a pie de obra, con suelos del terreno, fibra natural (gramíneas de lotes vecinos) picada a machete, y agua. El revoque se colocó a mano y luego se alisó con fratacho de madera; y finalmente luego de dos horas de secado se humedeció y alisó con presión con uso de llanas, para que la superficie final quedara apenas compactada y a la vez bastante lisa (para permitir pinturas o lechadas posteriores).

En el caso del muro de tierra vertida que se experimenta en el barrio de Nuevo Golf, Mar del Plata, se diseñó un prototipo a partir de la técnica que consiste en el colado de morteros de composición mixta en moldes móviles. Se construyó un muro de cerramiento exterior de $40 \mathrm{~cm}$ de espesor en el living-comedor de una vivienda existente, y se proyecta el sistema para un centro comunitario en actual construcción ("La Trinchera").

Los materiales que integran el mortero son suelos del lugar sin tamizar, piedras del terreno de diferente tamaño, escombros reciclados disponibles, un árido arenoso de diámetro mediano ( 0,5 a 1,5 $\mathrm{cm})$, cemento tipo Portland y agua. Las dosificaciones varían según el tipo de suelo y los tamaños de piedra disponibles, y del ancho del muro. Se probaron distintos morteros a partir de la disponibilidad de materiales existentes en el barrio, buscando probar dos porcentajes en volumen de cemento. Dos de los dosajes utilizados con resul- 


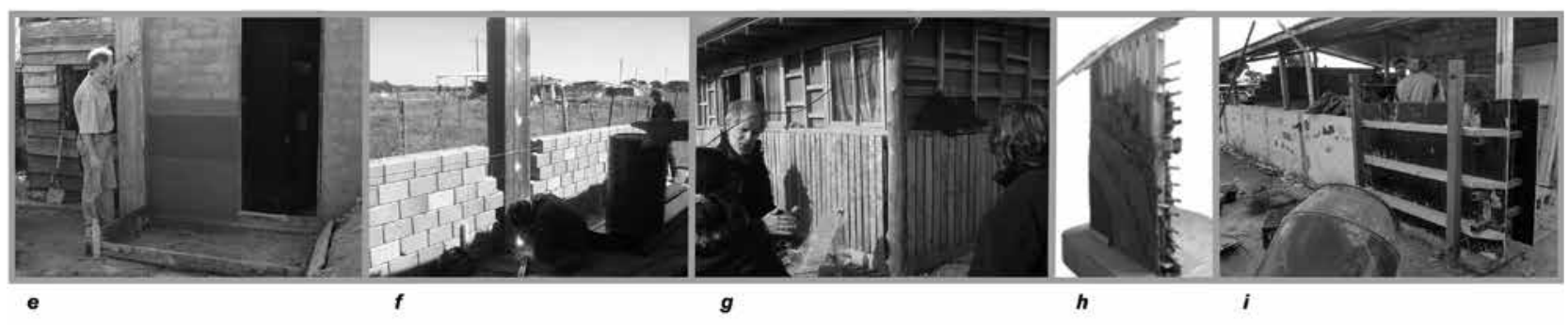

Figura 3. Elementos alternativos para construir muros. Prototipos con BTC en Bancalari (e) y Monte Terrabusi (f); de pallets con rellenos y revoques de tierra estabilizada con fibras naturales (g); y de tierra vertida en Nuevo Golf (h). Fuente: Rotondaro et al. (2013).

Tabla 3. Muros alternativos de BTC, entramado y tierra vertida.

\begin{tabular}{|c|c|c|c|c|c|}
\hline Prototipo & $\begin{array}{c}\text { Espesor } \\
(\mathbf{c m})\end{array}$ & $\begin{array}{c}\text { Materiales/ } \\
\text { morteros* }^{*}\end{array}$ & Técnica & $\begin{array}{c}\text { Diseñadores/ } \\
\text { autoconstructores }\end{array}$ & $\begin{array}{c}\text { Otros } \\
\text { aspectos }\end{array}$ \\
\hline $\begin{array}{l}\text { Muro de BTC en } \\
\text { Bancalari (e) } \\
9,5 \times 14 \times 29 \mathrm{~cm}\end{array}$ & 14 & $\begin{array}{l}9: 1 \text { a } 14: 1 \\
\text { suelo-cemento } \\
\text { (bloque y mortero) }\end{array}$ & $\begin{array}{l}\text {-Prensa } \\
\text { CINVA-RAM } \\
\text {-Mampostería }\end{array}$ & $\begin{array}{l}\text { Vecinos, arquitectos, } \\
\text { estudiantes }\end{array}$ & $\begin{array}{l}\text { Posible para } \\
\text { autoconstructores }\end{array}$ \\
\hline $\begin{array}{l}\text { Muro de BTC en } \\
\text { Monte Terrabusi (f) } \\
9,5 \times 14 \times 22 \mathrm{~cm}\end{array}$ & 14 & $\begin{array}{l}10: 1 \\
\text { suelo-cemento } \\
\text { (bloque y mortero) }\end{array}$ & $\begin{array}{l}\text {-Prensa } \\
\text { hidráulica } \\
\text {-Mampostería } \\
\end{array}$ & $\begin{array}{l}\text { Vecinos, arquitectos, } \\
\text { autoconstructores, } \\
\text { estudiantes }\end{array}$ & $\begin{array}{l}\text { Posible para } \\
\text { autoconstructores }\end{array}$ \\
\hline $\begin{array}{l}\text { Muro de pallets en } \\
\text { Monte Terrabusi } \\
\text { (g) }\end{array}$ & 18 & $\begin{array}{l}\text { Columnas y vigas } \\
\text { de madera, pallets, } \\
\text { relleno y revoques } \\
\text { con suelos arcillosos } \\
\text { y fibra vegetal } \\
\text { picada }\end{array}$ & $\begin{array}{l}\text {-Estructura } \\
\text { independiente } \\
\text {-Pallets rellenos } \\
\text { y revocados }\end{array}$ & $\begin{array}{l}\text { Vecinos, arquitectos, } \\
\text { estudiantes }\end{array}$ & $\begin{array}{l}\text {-Construcción } \\
\text { rápida } \\
\text {-Posible para } \\
\text { autoconstructores }\end{array}$ \\
\hline $\begin{array}{l}\text { Muro in situ de } \\
\text { tierra vertida en } \\
\text { Nuevo Golf }(\mathrm{h})\end{array}$ & 40 & $\begin{array}{l}\text { Mortero mixto } \\
1: 2: 4: 4: 6 \\
\text { cemento- } \\
\text { piedra-árido } \\
\text { mediano- } \\
\text { escombros-suelos }\end{array}$ & -Muro portante & Vecinos, arquitectos & $\begin{array}{l}\text { Construcción } \\
\text { rápida }\end{array}$ \\
\hline
\end{tabular}

*Porcentaje de partes en volumen.

tados satisfactorios fueron 10:1:3:3:1 y 12:1:4:1:2 (suelo sin tamizar: piedra: escombro: árido mediano: cemento).

Los moldes empleados son de multilaminado enchapado con melamina de $18 \mathrm{~mm}$ de espesor, vigas horizontales de madera de pino de 2" x 4" atornilladas, soportes verticales de caño estructural hueco de 2" x 4", y sujetadores de varilla de hierro roscada con doble arandela y tuerca. La dimensión interior libre del molde es de 0,40 m $\mathrm{x} 1,10 \mathrm{~m} \times 1,20 \mathrm{~m}$ para tramos rectos, y se rellena hasta la altura de 1 metro.

La fabricación del muro se realizó por tramos horizontales hasta completar tres paredes del ambiente (living-comedor), sobre un cimiento de hormigón. Se coló el mortero por capas sucesivas de unos 30 $\mathrm{cm}$ de alto, con ayuda de varillas de hierro para evitar que queden huecos o zonas mal rellenas. Se desmoldó luego de tres días para permitir su secado final a la intemperie. 


\section{Hornos y estufas domiciliarias de masa térmica con paredes y bóveda de adobe (Figura 4, Tabla 4)}

Los dos primeros prototipos se construyeron en San Martín (GBA) y en Monte Terrabusi (Mar del Plata) (Rotondaro et al., 2013).

Uno de ellos es el horno de tambor, compuesto por un tambor metálico de 200 litros con puerta, apoyado sobre paredes de mapostería de adobe y mortero de suelo y fibras naturales. Los muretes se continúan con los mismos componentes y recubren el tambor formando una bóveda de cañón corrido, a $8 \mathrm{~cm}$ de separación del tambor. Por debajo del tambor queda un espacio de unos $60 \mathrm{~cm}$ utilizado para el fuego directo y el cenicero. Los adobes y el mortero de asiento se fabricaron y secaron en el lugar (predio de Cobijo Urbano, Cooperativa Juntos Podemos Más, San Martín).

El otro prototipo fue construido en la pared exterior de la cocina de una vivienda existente, con doble acceso: al exterior a la cámara de fuego, y desde el interior para introducir y extraer las bandejas con alimentos. Para la base del horno se emplearon bloques cementicios de áridos de descarte (dos hiladas) y adobes con mortero de suelos y fibras naturales (cuatro hiladas); luego se apoyó el tambor sobre vigas metálicas apoyadas en las paredes de adobe y se construyó la bóveda de cañón corrido con iguales adobes y mortero.
Ambos modelos se revocaron a mano y fratacho, con un revoque de unos 1,5 cm de espesor con mortero muy mezclado durante varios días, compuesto por suelos arcillosos, fibra natural picada, estiércol de caballo y agua.

Un tercer caso (Nanami et al, 2014) es una estufa de doble combustión y alto rendimiento, construida con paredes de mampostería de adobe y ladrillos refractarios. El primer prototipo fue diseñado, construido y ensayado por un grupo mixto de investigadores y técnicos del CONICET, del INTI (Instituto Nacional de Tecnología Industrial) y de la Facultad de Arquitectura, Diseño y Urbanismo de la Universidad de Buenos Aires.

El modelo, denominado finalmente estufa SARA, se fabricó enteramente en el predio del INTI Migueletes, en San Martín, Buenos Aires. La estufa tiene $54 \mathrm{~cm}$ de ancho, $78 \mathrm{~cm}$ de largo y 104 $\mathrm{cm}$ de alto. Las paredes son de mampostería de adobes de $11 \mathrm{~cm} \times 11 \mathrm{~cm} \times 23 \mathrm{~cm}$ y tienen una hilada de ladrillos refractarios industriales a mitad de altura (piso de la segunda cámara de combustión). La cámara de la primera combustión (leña) está revestida con tejuelas refractarias, con el fin de mejorar su rendimiento. La estufa se revistió solamente con una lechada muy fina y plástica, a fin de evitar capas de revoques, compuesta por el mismo suelo natural con el que se fabricaron los adobes y su mortero de asiento.

La puerta es de hierro de chapa gruesa y marco de planchuelas L, y las chimeneas son de hierro

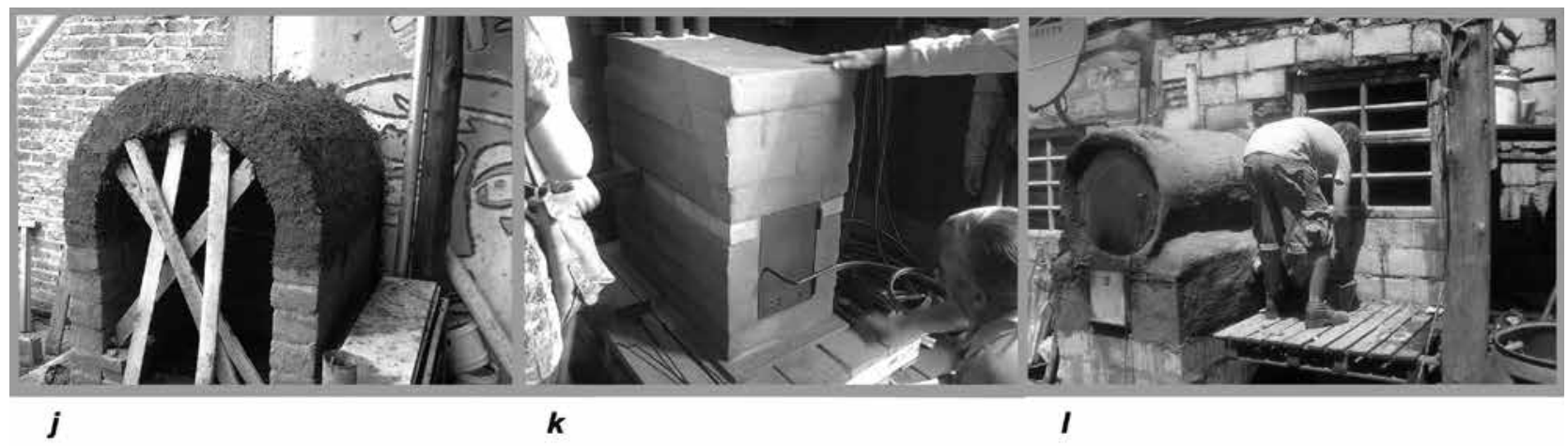

Figura 4. Elementos alternativos para cocinar y para aire caliente. Prototipos construidos en: San Martín (j) e INTI Migueletes (k) en Buenos Aires; y Monte Terrabusi (l) en Mar del Plata. Fuente: Rodolfo Rotondaro. 
Tabla 4. Elementos domiciliarios de masa térmica para cocinar y calefaccionar.

\begin{tabular}{|c|c|c|c|c|c|}
\hline Prototipo & $\begin{array}{c}\text { Dimensiones } \\
(\mathbf{c m})\end{array}$ & $\begin{array}{c}\text { Materiales / } \\
\text { morteros }\end{array}$ & Técnica & $\begin{array}{l}\text { Diseñadores / } \\
\text { constructores }\end{array}$ & $\begin{array}{c}\text { Otros } \\
\text { aspectos }\end{array}$ \\
\hline $\begin{array}{l}\text { Horno en San } \\
\text { Martín (i,j) }\end{array}$ & $\begin{array}{l}\text { Prof. } 89 \\
\text { Diám. } 57 \\
\text { Alto } 156\end{array}$ & $\begin{array}{l}\text { Tambor y puerta de } \\
\text { hierro, adobes, mortero } \\
\text { de suelos, fibras y } \\
\text { estiércol, chimeneas } \\
\text { metálicas }\end{array}$ & $\begin{array}{l}\text { Mampostería, } \\
\text { bóveda de } \\
\text { cañón corrido }\end{array}$ & $\begin{array}{l}\text { Vecinos, } \\
\text { arquitectos, } \\
\text { autoconstructores }\end{array}$ & $\begin{array}{l}\text { Posible para } \\
\text { autoconstructores }\end{array}$ \\
\hline $\begin{array}{l}\text { Horno en } \\
\text { Monte } \\
\text { Terrabusi (k) }\end{array}$ & $\begin{array}{l}\text { Prof. } 105 \\
\text { Diám. } 57 \\
\text { alto } 145\end{array}$ & $\begin{array}{l}\text { Tambor y puerta de } \\
\text { hierro, adobes, mortero } \\
\text { de suelos y fibras, arena, } \\
\text { chimenea metálica }\end{array}$ & $\begin{array}{l}\text { Mampostería, } \\
\text { bóveda de } \\
\text { cañón corrido }\end{array}$ & $\begin{array}{l}\text { Vecinos, } \\
\text { arquitectos }\end{array}$ & $\begin{array}{l}\text { Posible para } \\
\text { autoconstructores }\end{array}$ \\
\hline $\begin{array}{l}\text { Estufa de } \\
\text { adobe INTI / } \\
\text { CONICET (l) }\end{array}$ & $\begin{array}{l}\text { Ancho } 54 \\
\text { Largo } 78 \\
\text { Alto } 104\end{array}$ & $\begin{array}{l}\text { Adobes, arena, mortero } \\
\text { de suelos con fibra } \\
\text { natural picada, ladrillos } \\
\text { y tejuelas refractarias, } \\
\text { planchuela de hierro, } \\
\text { caños de hierro negro, } \\
\text { hierro galvanizado }\end{array}$ & $\begin{array}{l}\text { Mampostería } \\
\text { de adobes con } \\
\text { una hilada } \\
\text { de ladrillos } \\
\text { refractarios, } \\
\text { lechada de } \\
\text { revestimiento }\end{array}$ & $\begin{array}{l}\text { Arquitectos, } \\
\text { ingenieros, } \\
\text { técnicos }\end{array}$ & $\begin{array}{l}\text { Posible para } \\
\text { autoconstructores }\end{array}$ \\
\hline
\end{tabular}

negro, tres de 7,5 cm de diámetro, hasta un cajón de humo de hierro galvanizado, del cual sale al exterior un caño de hierro galvanizado de $15 \mathrm{~cm}$ de diámetro.

La eficiencia energética fue medida por el laboratorio de Energía del INTI, y superó el 65\%, dato a partir del cual se diseñó el manual de transferencia para su difusión masiva (INTI-CONICET, 2014).

\section{Revoques y terminaciones alternativas con técnicas de tierra} (Figura 5, Tabla 5)

Se experimentaron distintos revoques y terminaciones con empleo de suelos estabilizados con fibra natural, cal y cemento, con distinta participación de autoconstructores (Cacopardo, Cusán y Rotondaro, 2013, pp. 119-145). Se diseñaron y construyeron de acuerdo con la adherancia necesaria para diferentes sustratos de aplicación: muro de BTC de suelo-cemento, muro de adobe, y muro mixto de pallets rellenos con malla metálica.

En Bancalari (GBA) se estudió principalmente el uso de una sola capa de revoque, su mortero, el espesor y la técnica. Se trabajó con morteros de suelo-cal y de suelo-cemento-cal. Los suelos empleados fueron los mismos de los muros de BTC, tamizados con malla de 3 a $4 \mathrm{~mm}$ de abertura. La aplicación se realizó con la técnica tradicional: previa limpieza y humedecido de la pared, se rellenaron huecos y juntas mal rellenas, y se aplicó el mortero con auxilio de testigos de espesor, regla, plomada y nivel. La terminación del revoque se realizó con fratacho de madera humedecido, y los espesores variaron entre 12 a $18 \mathrm{~mm}$.

Se probaron además dos terminaciones directamente sobre la pared, una como pintura espesa con un mortero mucho más plástico que el de los revoques; y otra como bolseado manual, con trapos y gomaespuma. Los morteros fueron los mismos que los de los revoques, y los espesores resultaron de entre 0,3 a $0,6 \mathrm{~mm}$.

En el caso de las mamposterías de adobe se aplicaron dos terminaciones diferentes. En la estufa SARA (San Martín) se utilizó una lechada compuesta por el mismo suelo de los adobes tamizado con malla de 1,5 mm de abertura. Se aplicó a mano, previo humedecido de las paredes, rellenando pequeños huecos de los adobes y las juntas, con un espesor final de 2 a $3 \mathrm{~mm}$. En cambio en 


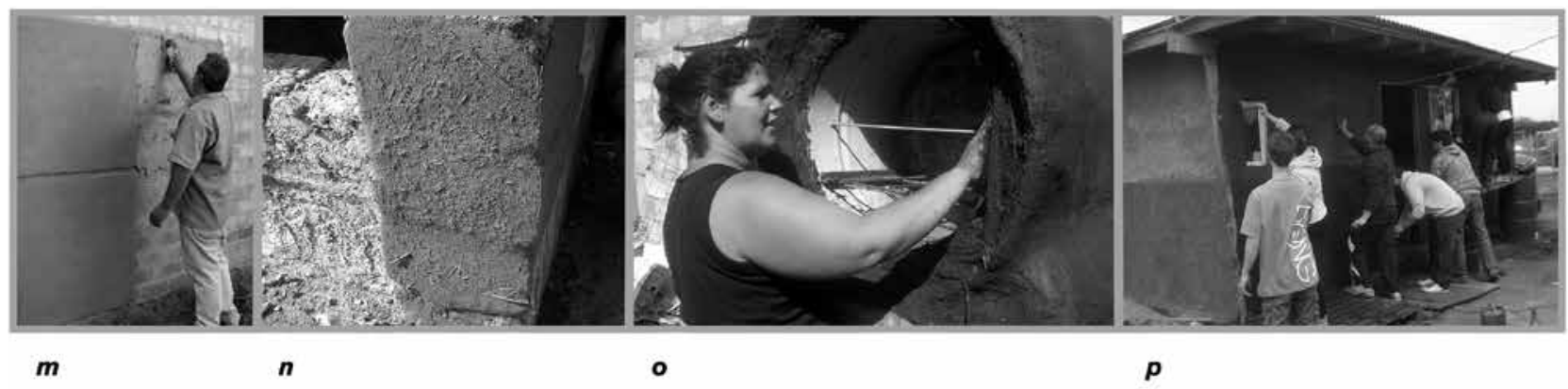

Figura 5. Elementos alternativos para revoques y terminaciones. Prototipos construidos en: Bancalari (m); San Martín (n) e INTI Migueletes (o), en Buenos Aires; Nuevo Golf (p) en Mar del Plata. Fuente: Rodolfo Rotondaro.

Tabla 5. Revoques y terminaciones de tierra alternativos.

\begin{tabular}{|l|l|l|l|l|l|}
\hline \multicolumn{1}{|c|}{ Prototipo } & $\begin{array}{c}\text { Espesor } \\
\text { (mm) }\end{array}$ & \multicolumn{1}{|c|}{$\begin{array}{c}\text { Materiales/ } \\
\text { morteros* }\end{array}$} & \multicolumn{1}{c|}{ Técnica } & \multicolumn{1}{c|}{$\begin{array}{c}\text { Diseñadores/ } \\
\text { autoconstructores }\end{array}$} & \multicolumn{1}{c|}{$\begin{array}{c}\text { Otros } \\
\text { aspectos }\end{array}$} \\
\hline $\begin{array}{l}\text { Revoques gruesos } \\
\text { en Bancalari (m) }\end{array}$ & 12 a 18 & $\begin{array}{l}9: 1 \text { a } 12: 1 \\
\text { suelo-cemento } \\
10: 1 / 2: 1 \\
\text { suelo: cemento:cal }\end{array}$ & $\begin{array}{l}\text { Una capa, por } \\
\text { paños cuadrados, } \\
\text { humedecido previo }\end{array}$ & $\begin{array}{l}\text { Vecinos, } \\
\text { arquitectos }\end{array}$ & $\begin{array}{l}\text { Posible para } \\
\text { autoconstructores }\end{array}$ \\
\hline $\begin{array}{l}\text { Revoque fino en } \\
\text { San Martín (n) }\end{array}$ & 2 a 3 & $\begin{array}{l}\text { Aplicación manual, } 1 \\
\text { suelo-cemento } \\
\text { humedecido previo }\end{array}$ & Arquitectos & $\begin{array}{l}\text { Posible para } \\
\text { autoconstructores }\end{array}$ \\
\hline $\begin{array}{l}\text { Revoque fino en } \\
\text { Mte. Terrabusi (o) }\end{array}$ & 4 a 6 & $\begin{array}{l}\text { Suelo arcilloso } \\
\text { y fibra natural } \\
\text { picada }\end{array}$ & $\begin{array}{l}\text { Aplicación manual, } \\
\text { con humedecido } \\
\text { previo }\end{array}$ & Autoconstructores & $\begin{array}{l}\text {-Construcción rápida } \\
\text {-Posible para } \\
\text { autoconstructores }\end{array}$ \\
\hline $\begin{array}{l}\text { Revoque grueso } \\
\text { en Mte. Terrabusi } \\
\text { (p) }\end{array}$ & 18 a 25 & $\begin{array}{l}\text { Suelo arcilloso } \\
\text { y fibra natural } \\
\text { picada }\end{array}$ & $\begin{array}{l}\text { Aplicación manual, } \\
\text { humedecido previo }\end{array}$ & $\begin{array}{l}\text { Vecinos, } \\
\text { arquitectos, } \\
\text { autoconstructores }\end{array}$ & $\begin{array}{l}\text {-Construcción rápida } \\
\text {-Posible para } \\
\text { autoconstructores }\end{array}$ \\
\hline
\end{tabular}

*Porcentaje de partes en volumen.

el horno de Monte Terrabusi (Mar del Plata), se aplicó a mano una lechada similar al mortero de asiento de los adobes pero con mayor plasticidad, tamizado más fino del suelo, y fibra natural picada muy corta, con un espesor de 4 a $6 \mathrm{~mm}$.

En el caso de los muros del prototipo con pallets (Monte Terrabusi), se aplicó a mano un revoque compuesto por suelos arcillosos locales tamizados y fibra natural picada, sobre malla metálica de gallinero (abertura de malla de $5 \mathrm{~cm}$ ). Previa colocación del revoque se humedeció el relleno de los pallets, de tal modo de evitar la fisuración por el secado rápido del revoque. El espesor promedio varía entre 18 y $25 \mathrm{~mm}$. La terminación se realizó con fratacho de madera, generando un alisado y relleno de pequeños huecos del mismo revoque.

\section{Conclusiones y comentarios finales}

El trabajo en territorio en los barrios y la aplicación de los métodos participativos, a lo largo de una década, han demostrado la factibilidad técnica y social del empleo de modelos constructivos alternativos basados en la tecnología de construcción con tierra, como una opción posible de desarrollar en contextos urbanos con población pobre.

En concordancia con los diagnósticos previos en cada contexto, y con la disponibilidad de recursos materiales y humanos, sumados al interés de los autoconstructores locales, los prototipos construidos muestran que algunas de estas alternativas podrían resultar apropiadas para su transferencia 
y réplica, con fuerte participación local. De hecho, en varios barrios hay demandas espontáneas de otros vecinos que participaron o tuvieron oportunidad de observar las innovaciones, con la idea de poder realizar mejoramientos similares en sus propias viviendas.

Otra observación importante a partir de los resultados obtenidos en territorio es que las técnicas y los procedimientos empleados han logrado, en general, un grado de aceptación popular, porque concuerdan con muchas de las lógicas de la "autoconstrucción tradicional" de los barrios. En especial, la organización de obra, los tiempos constructivos, los conocimientos básicos de albañilería de muchos de los autoconstructores, y las lógicas de mejoramiento en forma progresiva del hábitat domiciliario.

En relación con los costos económicos de los diferentes prototipos se observó que en general se obtienen costos menores (en todos los casos) comparados con los elementos similares de la construcción tradicional industrializada, y que estos costos son posibles en el contexto de esta población.

En el caso de los muros, considerando los costos de materiales industrializados empleados (cemento, cal, madera, hierros, alambre, clavos), los costos de transporte en algunos casos, y los costos estándares de los jornales de albañilería, los muros de adobe y de pallets rellenos y revocados fueron entre un 30 y un $40 \%$ más económicos comparados con los muros de ladrillo hueco y de bloques de hormigón revocados en ambas caras. En esta diferencia influyó notablemente la gratuidad de los materiales obtenidos localmente (suelos, fibras naturales, agua, piedras, escombros de obra, madera reciclada), la donación de áridos de Cantera Yaraví, y la participación de los autoconstructores y familias beneficiarias.

En el caso de los BTC fabricados y donados por la Fundación UOCRA (Mara del Plata), hubo costos mínimos de transporte, pero dado que los muros no necesitan ni mortero de asiento ni revoques, y además fueron autoconstruidos por la familia, el único costo económico fue el del cemento, con lo cual esta situación también ubicó a este prototipo dentro de las posibilidades del contexto barrial.

De igual manera, los costos económicos de los prototipos de contrapisos y pisos, y de revoques y lechadas de terminación, resultaron siempre por debajo de los costos de los mismos elementos constructivos tradicionales industrialilzados.

En cuanto a los prototipos de horno, resultaron prácticamente gratuitos en su costo económico, ya que únicamente se invirtieron gastos de materiales de herrería y soldaduras para las puertas, bandejas y chimeneas, siendo gratuita la construcción completa del horno. En el caso del modelo de estufa SARA con paredes de adobe, si bien fue necesario comprar los ladrillos y tejuelas refractarias y pagar jornales de herrería para la puerta y los conductos, se observó que dichos costos significan una diferencia de más del $40 \%$ si se los compara con el precio total de cualquier artefacto de calefacción similar del mercado formal (salamandras, estufas de hierro de simple y doble combustión).

El trabajo en territorio con los autoconstructores y sus familias demostró que el empleo de materiales naturales disponibles localmente (tales como suelos, fibras vegetales, estiércol de caballo, piedras y agua) y las combinaciones con materiales y elementos industrializados reciclados y nuevos (tales como pallets, hierro, vigas de madera, elementos metálicos, escombros, residuos de cantera de piedra), han posibilitado la generación de soluciones mixtas con empleo de técnicas de construcción con tierra, con una fuerte adaptación a cada contexto local. Esta situación se vio fortalecida por la continuidad en las capacitaciones y trabajos de campo, en tiempos aceptables, que permitió a los autoconstructores adquirir conocimientos básicos que posibilitan el uso y la réplica de algunas de las innovaciones.

También han demostrado que es necesario una asistencia técnica mínima en la producción de adobes y BTC, así como en la aplicación de los revoques con suelos estabilizados, en la terminación de los pisos y en la construcción de artefactos de cocción y calefacción. 
Con relación al desempeño de las mezclas entre los suelos naturales locales y los dos estabilizantes industrializados utilizados en los BTC y revoques (cal hidratada y cemento tipo Portland), los resultados demostraron ser satisfactorios en el $100 \%$, en cuanto a dureza en los BTC, y el $40 \%$ en cuanto a dureza y adherencia en los revoques. Tres de los seis porcentajes en volumen de cal hidratada y dos de los tres porcentajes en volumen de cemento empleados, están dentro de los dosajes habituales de las técnicas de construcción con tierra, en relación a los resultados de dureza y durabilidad registrados en el período evaluado (seis años).

Dado que la estrategia de la investigación tiene incorporadas importantes tareas de transferencia tecnológica, los resultados que se presentan en este trabajo evalúan principalmente una de las etapas de generación de tecnología con fuerte trabajo en territorio. En este sentido, uno de los factores que fortalece el trabajo de campo es la metodología con participación local, que se experimenta, se ajusta y se vuelve a diseñar a medida que se desarrollan las actividades. Este objetivo metodológico es sustancial para la investigación y tiene fundamento en el marco teórico de las tecnologías sociales. Considera indispensable la participación de autoconstructores y familias, para poder articular una red de alianzas socio-técnicas con una multiplicidad de actores públicos y privados.

En la Figura 6 se presenta una síntesis del modelo de gestión participativa y multisectorial utilizado:

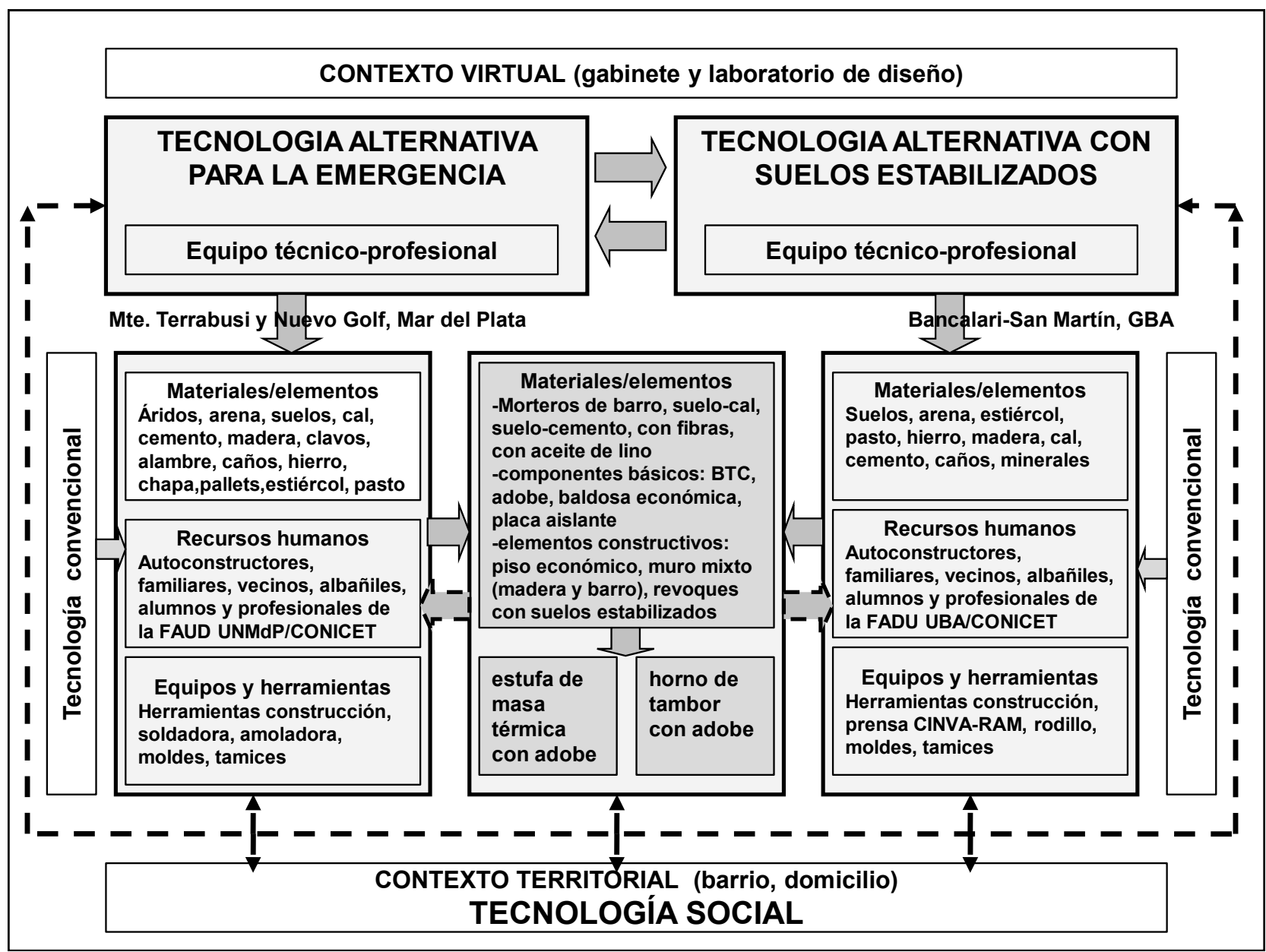

Figura 6. Estrategia y modelo de gestión. Alianzas multisectoriales para la generación de tecnología social en los territories barriales. Fuente: elaboración propia. 
La evaluación cualitativa de esta estrategia indica los avances obtenidos a partir del desarrollo de los prototipos experimentales en territorio, en el sentido de haber logrado una contínua y fuerte participación local de los pobladores y líderes barriales. Estos indicios apuntalan la factibilidad social y económica de algunas de las soluciones experimentales (tanto de gestión de tecnología como de innovaciones en el ámbito de la autoconstrucción asistida), y su proyección hacia etapas siguientes de autogestión y gestión asistida, buscando el mejoramiento progresivo de la calidad material e inmaterial del hábitat doméstico en estos barrios.

Por otra parte, se observó también que en la escala familiar las innovaciones son posibles. Es decir, los objetivos y los supuestos (hipótesis) de la investigación, orientados a la factibilidad social de los cambios tecnológicos, muestran resultados satisfactorios en cuanto a la aceptación local y a una posible réplica en una escala con mayor cantidad de pobladores y familias.

Los autores de este trabajo están convencidos de la importancia de promover el desarrollo de pequeñas producciones a escala doméstica de alternativas constructivas basadas en técnicas de construcción con tierra y mixtas, como se ha venido realizando en los barrios con los pisos, los revoques y los muros alternativos, en reemplazo o en combinación con los elementos similares de la construcción tradicional industrializada (bloques de hormigón, ladrillos cerámicos huecos, revoques cementicios, pisos de hormigón).

Sin embargo, y también como parte de la evaluación integral de los resultados de esta etapa de la investigación, han aparecido limitaciones comunes para el desarrollo de estas alternativas. Estas limitaciones están directamente relacionadas con la réplica de los prototipos (no es pareja ni total en ninguno de los barrios en cuestión), con los tiempos y la difusión necesarios para que sean mejor apropiados; y con la resolución de problemas propios de la complejidad de la pobreza estructural de la población. En este sentido, la continuidad del programa de investigación en los mismos territorios barriales garantizaría un período más prolongado, útil para poder evaluar distintas etapas y momentos del país, que puedan producir resultados más consolidados y procesos de mejoramiento progresivo en distintas viviendas, que permitan mostrar un cambio tecnológico real.

Los autores piensan también que el empleo de la tecnología de construcción con tierra podría permitir algunas ventajas, comparada con la tecnología de construcción tradicional industrializada. Estas ventajas estarían orientadas al ahorro energético domiciliario, a disminuir la contaminación ambiental, la quema de materiales fósiles, el consumo de agua y los costos económicos relativos de los elementos básicos de la vivienda autoconstruida.

\section{Referencias bibliográficas}

Aranda Jiménez, Y. G. y. Roux Gutiérrez, R. S. (2012). Comparativa de durabilidad entre BTC y tierra vertida. Construcción con Tierra, CT5, 115-126. Ciudad de Buenos Aires, Argentina: CIHE-FADU UBA.

Cacopardo, F., Cusán, M.I. y Rotondaro, R. (2013). Tecnologías sociales como un emergente territorial: aportes para un modelo de gestión del hábitat popular. Experiencia matriz para pilares de conexión eléctrica, Mar del Plata, Argentina. Revista Cuaderno Urbano-Espacio, Cultura, Sociedad, Vol. 14, №14, pp. 119-145. Universidad Nacional del Nordeste. Resistencia, Argentina: Nobuko-EUDENE. Disponible en: [http://revistas.unne.edu.ar/index.php/crn/ issue/ view/92/showToc].

Dagnino, R., Cruvinel Brandão, F. y Tahan Novaes, H. (2004). Sobre o marco analítico conceitual da tecnologia social. En: Tecnología Social. Uma estratégia para o desenvolvimento. . Río de Janeiro, Brasil: Fundação Banco do Brasil. Disponible en: [http://www.mom.arq.ufmg. br/mom/18_ref_capes/arquivos/arquivo_110. pdf]. 
INDEC (2002). Censo Nacional de Población, Hogares y Viviendas 2001. Ciudad de Buenos Aires, Argentina: Ministerio de Economía, Instituto Nacional de Estadísticas y Censos, Gobierno de Argentina. Disponible en: [https:// www.indec.gov.ar/ nivel4_ default.asp?id_ tema_1=2\&id_tema_2=41\&id_tema_3=134].

INDEC (2011). Censo Nacional de Población, Hogares y Viviendas 2010. Ciudad de Buenos Aires, Argentina: Ministerio de Economía, Instituto Nacional de Estadísticas y Censos, Gobierno de Argentina.Disponible en: [https:// www.indec.gov.ar/nivel4_default.asp? id_tema _1=2\&id_tema_2=41\&id_tema_3=135].

INDEC (2013). Encuesta Permanente de Hogares (2013, 2014). Incidencia de la pobreza y de la indigencia. Resultados del primer semestre de 2013. Ciudad de Buenos Aires, Argentina: Ministerio de Economía, Instituto Nacional de Estadísticas y Censos, Gobierno de Argentina. Disponible en: [https://www.indec.gob. ar/nivel4_default.asp?id_tema_1=4\&id_tema_ $2=27 \&$ id_tema_3=64].

INTI-CONICET (2014). Manual estufa SARA. San Martín, Argentina: Ediciones INTI. Disponible en: [www.estufasara.inti. gov.ar].

Kessler, G. y Di Virgilio, M. M. (2008). La nueva pobreza urbana: dinámica global, regional y argentina en las últimas dos décadas. Revista de la CEPAL, 95, pp. 32-50. Santiago, Chile: CEPAL.

Nanami, A., Lambert, A., Tejerina, D., Dabbah, F., Mario Ogara, M. Tedesco, M. y Hugón, N. (2014). SARA. Estufa social argentina de alto rendimiento. Manual para autoconstructores. San Martín, Argentina: INTI-CONICET-UBA.
Disponible en: [https://www.inti.gob.ar/publicaciones/descargac/545.

Neves, C. M. M., Faría, O. B., Rotondaro, R., Cevallos S. P. y Hoffmann, M. (2009). Selección de suelos y métodos de control en la construcción con tierra. Prácticas de campo. Cuaderno 2009, pp. 15-26. Red Iberoamericana PROTERRA. Disponible en: [http://www. redproterra.org].

Pelli, V. S. (1994). ¿Cómo entendemos la pobreza? Las ONG en la construcción de la ciudad. Seminario Internacional "La ciudad para todos", Programa Arraigo, Buenos Aires. Módulo: Formas participativas de la gestión habitacional. Argentina: Facultad de Arquitectura, Urbanismo y Diseño, Universidad Nacional de Mar del Plata.

Pelli, V. S. (2007). Habitar, participar, pertenecer. Buenos Aires, Argentina: Ed. Nobuko.

Rotondaro, R, Patrone, J. C. y Schicht, A. (2006). Diseño de pisos y revoques con empleo de tierra tosca estabilizada. Construcción con Tierra, 2, pp. 56-67. Buenos Aires, Argentina: CIHE-FADU UBA.

Rotondaro, R. y Cacopardo, F. (2013). Construcción con tierra y tecnologías sociales. Modos de gestión participativa en la materialidad de la vivienda. Horizontes Revista de Arquitectura, 6, pp. 16-22. Oaxaca de Juárez, México: Nuevos Horizontes para la Arquitectura de las Comunidades A.C. Disponible en: [http://horizontes18. com/revista-horizontes].

Thomas, H. (2009). De las tecnologías apropiadas a las tecnologías sociales. Conceptos/Estrategias/ Diseños/Acciones. IV Seminario Iberoamericano de Ciencia y Tecnología. Córdoba, Argentina: AVE CEVE-CONICET. 